\title{
La subjetividad al rescate del sujeto histórico en la investigación educativa ${ }^{1}$
}

\author{
Ángela María Cadavid Marín ${ }^{2}$
}

\begin{abstract}
Resumen
Generar conocimiento a partir de la propia subjetividad para interpretar los significados de los acontecimientos personales que describen y construyen lo existente, como un escenario emergente de la conciencia de los sentidos de realidad que viven los maestros, permite que lleven a cabo procesos de auto reflexión, donde sea posible problematizar los sucesos que en el ámbito educativo se presentan actualmente. En estas auto reflexiones participa el sujeto que relata su experiencia vital, identificando el contexto de sus comprensiones, para ubicar en un hoy los sucesos narrados y sus sentidos y para participar de manera activa en la construcción de futuros deseables que favorecen construcciones intersubjetivas de los actores que intervienen en las relaciones peda-gógicas en las instituciones educativas. Palabras claves: Huellas vitales, prácticas pedagógicas, experiencia, subjetividad, transformación, maestro, movilización y escuela.
\end{abstract}

\section{The subjectivity to the rescue of the historical subject in educational research}

\begin{abstract}
Generating knowledge from people's own subjectivity to interpret the mea-nings of personal events that describe and build what is already there, as an emerging stage of consciousness of the senses of reality experienced by teachers, allows to carry out self-reflective processes where it is possi-ble to problematize the ongoing events in the educational setting. In these self-reflections participates the subject who expresses his vital experience, identifying the context of his understandings, to locate in our present the narrated events and his senses and to be able to participate actively in the construction of desirable futures which favor intersubjective constructions from the people who intervene in pedagogical relationships in educational institutions.
\end{abstract}

Keywords: Vital traces, pedagogical practices, experience, subjectivity, transformation, teacher and school mobilization.

1 Recibido: 07 de agosto de 2016. Aceptado: 29 de noviembre de 2016.

2 Ángela María Cadavid Marín. Estudiante del Doctorado en Ciencias de la Educación: Pensamiento Educativo y Comunicación en la Universidad Tecnológica de Pereira; Magíster en Educación Docencia; Docente Investigadora de la Facultad de Ciencias Sociales y Humanas de la Universidad de Manizales, Instituto Pedagógico. Correo electrónico: acadavid@umanizales.edu.co 


\section{La subjetividad al rescate del sujeto histórico en la investigación educativa ${ }^{3}$}

\section{Yo, ser humano, nacido en el mundo social y que vivo mi existencia cotidiana en él, lo experimento como construido alrededor del lugar que ocupo en él, como abierto a interpretación y acción, pero siempre en referencia a mi situación real biográficamente determinada.}

(Schütz, 1995, p.45)

El contexto educativo actual viene transformándose, lo que genera interrogantes respecto de lo que emerge en los espacios educativos. De esta manera, se empieza a explorar para llegar a una instancia reflexiva y de acción frente a estos sucesos y problemáticas que se presentan y le dan un nuevo significado a la escuela. Y para ello, se busca ir más allá del día a día del maestro, para descifrar las simbologías que se perciben en su realidad, desde donde se pueden encontrar nuevas formas de relación y orientación al otro. “...en la construcción de la personalidad tienen una importancia capital las relaciones humanas" (Fernández, 2005, p.206).

De todo esto, empieza a interesar la identidad del sujeto maestro plasmada en el tiempo y cómo lo transitado hasta ahora ha logrado tener repercusiones en su actuar, en su ser como maestro; se piensa en la importancia de reconocer lo hecho y el porqué de ello. La relevancia de reconocerse a sí mismo si quiere ser alguien con otro alguien y ser consciente

3 Este artículo de reflexión se produce a partir del Macroproyecto de investigación "Prácticas pedagógicas y Huellas vitales", adscrito a la Maestría en Educación desde la Diversidad (Centros Tutoriales Pasto y Popayán) de la Universidad de Manizales, con varios proyectos que lo soportaron. Estudio realizado durante el período 2013-2016, cuya investigadora principal es la autora del presente texto. que ese alguien requiere que impacte en su formación rompiendo paradigmas y dejando huella, de tal manera que logre discernir en el hoy y a partir de él considerar e indagar limitantes e infinitos. En este sentido, Perreneud (1994, citado por Barbosa, 2014, p.78), afirma: "La práctica reflexiva es un trabajo, que demanda un cierto método y una cierta formación. Se trata de instalar una "rutina de cambio"'.

Asimismo, la importancia de reconsiderar su tarea y repensar la importancia de indagar sobre el porqué de su quehacer, del para qué de sus acciones, del cómo está articulando sus procesos y del dónde se está ubicando para comprender el contexto donde se desenvuelven. "Los docentes debemos comenzar por hacer consciencia y reflexión (cuestionando lo que estamos haciendo) sobre la forma como enseñamos y la de como deberíamos enseñar y a partir de ellas construir y direccionar la actitud y las prácticas pedagógicas" (Vega, 2009, p.192). En esta perspectiva, el maestro se ha de comprometer con la transformación de la realidad social que está emergiendo. “...la transformación de la vida cotidiana, de las relaciones y circunstancias de los hombres, no es anterior ni posterior a la transformación política y económica, sino simultánea con ella" (Heller, 1985, p.12).

Al respecto, Ortega y Gasset (1941, p.2) menciona que "la vida no es algo que se nos otorga, sino que es preciso descubrir"; lo que significa que todos los sujetos tienen la capacidad para elegir la manera más adecuada de explorar su vida y su quehacer; en este sentido, el maestro no está obligado a nada y al mismo tiempo obligado a todo; sin embargo, esta capacidad de elección pierde toda fuerza si desconoce la manera de relacionarse consigo mismo, con los otros, con otras culturas, con el mundo en general, con todo lo que le es próximo y lo que le es ajeno. En consonancia con lo anterior, Boivin, Rosato y Arribas (1989), afirman que "El Nosotros no sólo se apropia de algo sino que, además, participa activa- 
mente y a veces de modo determinante en la conformación de los atributos del 'otro'” (p.104).

En este orden de ideas, se ha de hacer consciente que su potenciación no la logrará defendiendo su individualidad, sino por el contrario, en el contacto con otros al enriquecer su propia vida, llevando este acto a un medio colectivo e histórico en donde necesariamente ha de ser alguien productivo, crítico, activo y asumiendo su naturaleza de ser sensitivo, corporal y social, lo que a la vez le permitirá organizar su pensamiento, su lenguaje y sus acciones en la misma medida que concretiza la cimentación de una identidad propia. "Tal identidad se construye en y desde la complejidad, asumiendo el carácter racional y relativo de su autonomía, de la implicación del significado de su individualidad y de sus diversos vínculos con el entorno" (Bernal, 2002, p.11).

Desde los anteriores planteamientos, la presente experiencia investigativa indagó sobre aquellas huellas vitales que han permanecido en el tiempo, que pasan inadvertidas y desapercibidas y que surgen de circunstancias y sucesos representativos durante el tránsito de los sujetos maestros por su mundo, lleno de interacciones y nexos. "Todo ser humano es una lección para otro, un texto abierto a la posibilidad de inventar nuevas realidades" Madriz (2004, p.7). De esta manera, interrogarse acerca de las situaciones, imaginarios y relaciones que se suceden en la vida de las personas desde su infancia y que configuran su subjetividad, es lo que constituye sus huellas vitales. La mayoría de estas experiencias se manifiestan ausentes en la cotidianidad, pero se hacen evidentes en algún momento por medio de la evocación.

Estudiar la subjetividad docente no es un mero ejercicio frívolo de intelectuales, es un lazo vinculante $y$ necesario del análisis de las posibilidades o imposibilidades de movilización social de este colectivo, de unas prácticas políticas, por ahora un tanto amnésicas, o tal vez solo disfónicas. (Robayo, 2015, p.232)

Así las cosas, Zemelman (1996) propone una subjetividad que deviene en lo social desde lo individual y colectivo donde se articulan la memoria, la cultura, la conciencia, la voluntad y la utopía; desde las cuales emerge la historicidad social que da sentido al presente y potencia el futuro.

Siempre que no se aborde con criterios reduccionistas, [la subjetividad] representa una situación de confluencia de planos de realidad en que se puede rastrear cómo desembocan los microprocesos (por ejemplo de índole psicológica) así como la apertura hacia ámbitos sociohistóricos que se caracterizan por ser inclusivos de otros planos que pueden constituir el contexto particular del sujeto concreto que interese estudiar. (p.99)

Cuando se habla de huella vital, se habla de un sello, de una marca de vida con una impronta de época que vincula al sujeto de distintas maneras con el tiempo; es decir, partir del reconocimiento y comprensión de la historia personal, lo que permite determinar las huellas vitales desde las cuales se despliegan sus sentires, sentimientos y vivencias, para dar miradas que permitan potenciar futuros que brinden aportes significativos en aras de la construcción y reconstrucción permanente de los aconteceres pedagógicos de la escuela, propendiendo por una educación que los lleve a asumirse como sujetos activos en la sociedad, repensando y orientando sus acciones pedagógicas hacia procesos innovadores y de creación en los espacios educativos, en pro de las posturas y de los imaginarios de los estudiantes que se encuentran en las aulas hoy en día. "Los procesos de innovación dependen en última instancia de la actitud, compromiso y saber hacer del docente" (De Miguel, 1991, citado en De La Torre, 1997, p. 65). 
Indagar sobre estas marcas generacionales, permite a los sujetos de la educación volver a pensar en sí mismos como protagonistas de los procesos educativos. "El hombre se enriquece como individualista de su experiencia social, a la vez que la sociedad se alimenta de la capacidad de los hombres para asumir la condición de sujetos protagónicos" (Zemelman, 2012, p.235).

Es importante reconocer las huellas vitales, identificarlas, hacerse consciente de su existencia, para contextualizarlas, reconfigurarlas y aún más superarlas, en cuanto se indagan, se confrontan y se comprenden para potenciarlas como generadoras de la revitalización de las relaciones pedagógicas entre los sujetos de la educación.

En este sentido, dichas huellas vitales son sellos y evocaciones individuales, pertenecen a un sujeto en particular, resultado de su cotidianidad; las cuales suscitan algún efecto en la vida de cada sujeto, en su desenvolvimiento social, familiar, escolar, cultural, laboral, entre otros. “...solo una parte muy pequeña de mi conocimiento del mundo se origina dentro de mi experiencia personal. En su mayor parte es de origen social..." (Schutz, 1995, p.44). Hacen parte de lo que cada sujeto es hoy en día, su manera de razonar, de entender, de comportarse, de opinar; sus motivaciones, las construcciones que ha elaborado para definir su rumbo en la vida, en fin, todo aquello que lleva guardado en su memoria. Todo aquello deja una impronta en el sujeto para definir su forma de actuar, de pensar y de ser en todas las actividades en donde interviene.

Apostar en la investigación por las huellas vitales, hace necesario hacer evidente la relevancia de la historia de vida de los sujetos para reconocer cómo lograron transformaciones en sus prácticas pedagógicas, a partir de la indagación de su realidad tanto familiar, como social, cultural, religiosa y escolar para develar qué marcas dejaron su impronta para llegar a ser lo que son y están siendo en la actualidad.

De esta manera, esta investigación como punto de partida de la indagación, reconoce que cada estudiante y cada maestro traen consigo una historia personal y social. En la actualidad, el asunto acerca del retorno del sujeto de la educación, se hace ineludible, en tanto este necesita re-pensarse, restituirse, verse, sentirse e historizarse de manera urgente, para comprender lo que le sucede en su realidad: sus vivencias, sus imaginarios, sus tradiciones, sus interacciones, sus costumbres y sus perspectivas; lo que significa arraigo a una historia no solo individual, sino colectiva.

Para lograrlo, el sujeto de la educación parte de su historicidad, para darle sentido y significado a sus posibilidades humanas, lo que le permitirá reconocerse como un sujeto con discernimiento, que se piensa, se reflexiona y actúa frente a las contingencias que se presentan en su día a día. Es decir, cada sujeto tiene la facultad de re-valorar su propia historia, visibilizando su mundo, haciendo lectura de época de manera única y singular. Esta evocación traerá consigo su recuperación en expresión de múltiples posibilidades e interacciones, en tiempos y espacios particulares, para, a partir de su experiencia, comprender quién es y para qué está en este mundo.

Pensamos en "las cosas pasadas"; las pensamos, las reavivamos, las somos, pero en realidad, no son "cosas" lo que la memoria recupera; recupera las "palabras" engendradas por la "imagen" de las cosas que, "pasando por los sentidos", imprimieron en el alma como huellas. (Xirau, 1985, como se cita en Burbano y Cortez, 2013, p.19)

La historicidad es fundamentalmente la posibilidad que tiene el sujeto de reconocerse en sus propias circunstancias, en su cotidianidad, en su experiencia de vida y la posibilidad que tiene como sujeto de trascender esas circunstancias en la 
cotidianidad y potencializarse. Historicidad es esa trascendencia que da la noción de tiempo presente y proyecto hacia la potenciación del presente como futuro.

Es decir, trascender las propias circunstancias, cotidianidades y experiencias de vida, es cuando el sujeto se da cuenta que existe una noción de tiempo presente, pero el tiempo presente en términos de tiempo generacional o de época. La relación pedagógica como una relación entre generaciones, es donde se encuentra la brecha. "Sabemos bien que las experiencias humanas, en un primer momento, son vividas, con toda la carga emocional que supone y, en un segundo momento, son enunciadas, mediante los juicios de valor que hacemos sobre ellas" (Martínez y Carreño, 2014, p.157).

En este contexto, se ha de considerar que el sujeto de la educación construye su presente desde el reflejo de su historia de vida, por lo que muchas de las actuaciones del presente están inmersas en las vivencias que antaño viviera y que a la postre son el insumo para la construcción de su realidad. Es así, como construir nuevas realidades, se da en el establecimiento de los nexos entre pasado y presente para potenciar futuros que brinden aportes para mejorar las prácticas en pro de las posturas y de los imaginarios de los sujetos en su realidad inmediata.

\section{...el quehacer fundamental del}

maestro en el encuentro con los estudiantes y la comunidad dentro de un contexto sociocultural, como ese espacio donde se generan procesos de interacción y reflexión en torno a problemas, saberes $y$ cuestionamientos, como elementos dinamizadores y transformadores del acontecer diario del maestro. Cobos (2000)

Es así como, la construcción de realidad del sujeto conlleva considerar sus vivencias, en donde surge la necesidad de situarse en un momento histórico que lo lleve a empoderarse y a legitimarse para estar, ser y actuar en espacios específicos de realidad, esto deviene en la configuración de una postura reflexiva como sujeto que comprende su mundo y el lugar que allí ocupa. "Cuanto más me vuelvo sobre mi infancia distante, tanto más descubro que siempre tengo algo que aprender de ella" (Freire, 2005, p.31).

Se ha de considerar, a partir de lo anterior, que todos los sujetos son portadores de una historia y forjadores de un porvenir, constantemente avocados a vivenciar miles de experiencias que redundan sobre sus actitudes y procederes, lo que les permite hacer de su existencia un llamado a la reflexión y autoevaluación permanente, cuyo resultado se verá en sus obras, pensamientos y sentimientos concretos.

Todo este proceso, le permitirá al sujeto su conquista, su restitución, su reconocimiento, su retorno, a través del lenguaje para significar sus pensamientos, necesidades, valores, miradas, actitudes, emociones, alteridades, convicciones y afectaciones frente al mundo de la vida. El lenguaje reconstruye al sujeto, en tanto en el ejercicio de evocar y rememorar sus experiencias e interacciones, logra identificar lo que es, quién es y cómo ha sido esa construcción de su realidad, que no solo es individual, sino colectiva.

De esta manera, un sujeto con conciencia histórica, no solo acude a ella para sumergirse en su pasado, sino para develarse, para re-encontrarse consigo mismo, para fijar su mirada en el propio pasado evitando así el olvido de sí mismo, de sus atributos, significaciones y singularidades, para sorprenderse en su re-descubrimiento, en cuanto ha dejado de ser, se ha cosificado, ha abandonado toda huella de sensibilidad, perdiéndose en lo banal. En este sentido, recuperar su historia, no sólo lo facultará para reconocerse dentro de una realidad, sino que además le brindará la posibilidad de estremecerse, emocionarse y reivindicarse con su existencia en un sinnúmero de relaciones (con el otro y con lo otro). 
Por lo tanto, la subjetividad se va modificando por las cargas de emociones y sentimientos que en los diversos entornos (familiares, escolares y comunitarios) va abarcando el sujeto; cabe aclarar, que no todas esas cargas son activadas o recibidas en la misma intensidad o al mismo tiempo; todo dependerá en gran medida de la relevancia dada a las circunstancias, a los sujetos con los cuales se ha involucrado y a la trascendencia de acuerdo con la época y contexto en que han surgido (Heller, 1985).

A partir de los planteamientos epistémicos descritos, para la investigación, la narrativa como estrategia metodológica permite acercarse a la subjetividad de los sujetos de la educación, develando las huellas vitales que han dejado aquellas vivencias significativas y que lograron impactar en su forma de pensar y de ser, contribuyendo a forjar su identidad. La conciencia histórica se vuelve precisamente conciencia de nuestra relación con el tiempo presente a la luz de la marca o sello generacional.

Por lo tanto, los resultados en este contexto investigativo, no pretenden presentar una postura única, sino por el contrario, posibilitar procesos de introspección para que los maestros ahonden en el tema y se ocupen por revitalizar sus prácticas pedagógicas. "...los modos de la acción cotidiana, ya sean intelectuales o materiales- prácticas en plural, que responden a una lógica táctica, mediante las cuales el ser humano configura su experiencia como individuo y como comunidad construyendo cultura" (Campo, p. 42).

Para terminar, desde los planteamientos desarrollados, se presenta la necesidad de rescatar al sujeto maestro en la complejidad de sus vivencias generacionales y a partir de la construcción de sentido desde los acontecimientos representativos de la comunidad, lo que lleva a la producción colectiva de conocimiento y a la configuración de nuevas formas de pensar y crear la educación y que ayudan a constituir su identidad en dominios tanto corporales, como mentales, afectivos, colectivos y culturales, leídos en situaciones determinadas, las cuales convergen en su subjetividad y se constituyen en huellas vitales como vestigios perennes que afectan, fundan y dan significado a su propia existencia, a partir de acontecimientos representativos.

Al tenor de estas apreciaciones, es necesario adentrarse en el mundo de los sujetos de la educación para, a partir de un proceso dialogante, dar cuenta de la configuración de sus subjetividades, donde las historias y experiencias de vida permitan develar esa singularidad en relación con el otro, otorgando una significación profunda a las cuestiones humanas.

Cuando el sujeto construye su historia cuenta con un pasado, un presente y un futuro, pero la historia se lee desde el presente, en tanto del pasado vienen siendo las condiciones de emergencia de ese presente; y el futuro, la potencia del presente; no es algo que se está esperando, sino que se va elaborando desde este presente. El presente es la articulación de condiciones de emergencia, de posibilidad, de actualidad.

En este despliegue, se potencia un sujeto, que desde su subjetividad es una voz colectiva, es una voz de época y en ese sentido su testimonio es lo que alienta a la acción colectiva para mejorar las situaciones, en este caso, de las relaciones pedagógicas entre generaciones. 


\section{Bibliografía}

Barbero, Martín (1998). Jóvenes: des-orden cultural y palimpsestos de identidad. En: Viviendo a toda. Jóvenes, territorios culturales y nuevas sensibilidades. Bogotá, Siglo del Hombre Editores.

Barbosa, M. (2014). Aprendizaje y métodos de docencia avanzada. Madrid: ACCI.

Bernal, A. (2002). El concepto de "Autorrealización" como identidad personal: una revisión crítica. Cuestiones pedagógicas (16), 11-24. En: http://institucional.us.es/revistas/ cuestiones/16/art_1.pdf

Boivin, M; Rosato, A. y Arribas, V. (1989). Constructores De Otredad: Una introducción a la antropología social y cultural. ( $1^{\mathrm{a}} \mathrm{ed}$.). Buenos Aires: EUDEBA.

Bruner, J. (1988). Realidad mental, mundos posibles. Barcelona: Gedisa.

Burbano Cadena, Yojanna y Cortez Lagos, Isabel. (2013). Prácticas pedagógicas y emociones. En: http://ridum.umanizales.edu.co:8080/xmlui/bitstream/handle/6789/790/Yojanna_Burbano_Cadena_2013.pdf?sequence $=3$

Campo, R. \& Restrepo, M. (2002). La docencia como práctica. El concepto, un estilo, un modelo. Bogotá: CEJA.

Cobos, M.E. (2000). La práctica pedagógica, una mirada desde la investigación. En Forero, G. (comp.). Nuevos Horizontes Pedagógicos. Tunja: Universidad Pedagógica y Tecnológica de Colombia.

De La Torre, S. (1997). Innovación Educativa: I. El proceso de innovación. Madrid: Dykinson, S.L.

Fernández, M.R. (Diciembre de 2005). Más allá de la educación emocional. La formación para el crecimiento y desarrollo del profesorado. PRH como modelo de referencia. Interuniversitaria de Formación del Profesorado. En: http

Freire, P. (2005). Cartas a Cristina. Reflexiones sobre mi vida y mi trabajo. México: Siglo XXI Editores.

González González, M A. (2014). Miedos y olvidos pedagógicos. Rosario: Homo Sapiens.

Guarín, G. (2015). Acción política colectiva de las políticas de la soledad del yo a las políticas del nosotros en la diversidad. Manizales, Colombia: Universitaria.

Heller, A. (1985). Historia y vida cotidiana. Aportación a la sociología socialista. México: Grijalbo

Madriz, G. (2004). ¿Quién eres?... ¿Quién soy?. La autobiografía en el relato de lo vivido. Filosofía. (31), 1-8.

Martínez, M. y Carreño, P. (2014). Pensar la educación con Guillermo Hoyos-Vásquez. Magis 6(13), 153-160. En: http://revistas. javeriana.edu.co/index.php/MAGIS/article/ viewFile/8795/pdf

Ortega y Gasset, J. (1941). Historia como sistema. Madrid: Alianza Editorial. En: http:// biblio3.url.edu.gt/Libros/his_com.pdf

Robayo, A. (2015). Subjetividades docentes en la universidad. Colombiana de Educación. (68), 229-263.

Ricoeur, P. (2006). Del texto a la acción. Buenos Aires, Fondo de Cultura Económica.

Schütz. A. (1995). El problema de la realidad social. Buenos Aires: Amorrortu editores.

Serna, J. (1990). La reconquista de la subjetividad. Pereira, Colombia: Gráficas Olímpica.

Vargas, G. (2006). Pensar sobre nosotros mismos. Introducción fenomenológica a la filosofía en América Latina. Bogotá, Colombia: San Pablo.

Vega, A. (2009). Integración de alumnos con necesidades educativas especiales: ¿Existe coherencia entre el discurso y las prácticas pedagógicas ejercidas por los profesores básicos? Estudios Pedagógicos 35(2), 189-202. En: http://www. scielo.cl/scielo.php?script=sci_arttext\&pid $=$ S0718-07052009000200011

Zemelman, H. (1996). Problemas antropológicos y utópicos del conocimiento. México: El Colegio de México.

Zemelman, H. (2011). Los horizontes de la razón. España: Arthropos.

Zemelman, H. (2012). Subjetividad y realidad social. En Piedrahita, C., Díaz, A. \& Vommaro, P. (comps.). Subjetividades políticas: desafíos y debates latinoamericanos, 235-246.En: http://biblioteca.clacso.edu. ar/clacso/coediciones/20130218032232/ Subjetividadespoliticas.pdf 